\title{
Über den Wassergehalt des Schweineschmalzes und anderer Fette.
}

\author{
Von \\ K. Fischer und W. Schellens ${ }^{1}$ ). \\ Mitteilung aus dem Chemischen Laboratorium der Auslandsfleisch- \\ beschaustelle Bentheim.
}

Im vorigen Jahre ist von Polenske ein Verfahren $z u$ einer einfachen und sicheren Bestimmung des Wassergehaltes im Schweineschmalz veröffentlicht worden ${ }^{2}$ ), das von der Beobachtung ausgehend, daf geschmolzenes Schweineschmalz sich beim Abkühlen um so eher trübt, je mehr Wasser es enthält, im wesentlichen auf der Bestimmung der Trübungstemperatur, die bei gleichem Wassergehalt in fast gleicher Höhe liegen soll, beruht.

Durch Versuche stellte Polenske zunächst fest, daß wasserfreies Schmalz bei $96^{\circ}$ etwa $0,45 \%$ und bei $42^{\circ}$ etwa $0,15 \%$ Wasser löste. Innerhalb dieser Grenzen setzte Polenske zu vier verschiedenen vorher getrockneten Schmalzen wechselnde Mengen Wasser und bestimmte dann nach einem genau beschriebenen Verfahren die Trübungstemperatur, die bei allen vier Proben bei steigendem Wassergehalt ziemlich in denselben Grenzen ermittelt wurde.

Am Schlusse seiner Arbeit führt nun Polenske aus, daf es im Sinne seiner Ausführungen sein würde, wenn sein Verfahren möglichst vielfach einer Nachprüfung unterzogen und so zu Erörterungen über die Frage Anlaß geben würde. Das Verfahren ist hier nun in eingehendster Weise nachgeprüft worden und hat, um das gleich vorweg zu erwähnen, im großen ganzen zu denselben Zahlen geführt, wie sie von Polenske ermittelt worden sind. Die von uns gewonnenen Ergebnisse und gemachten Beobachtungen sollen nachstehend mitgeteilt werden.

I. Schweineschmalz.

Zur Nachprüfung des Verfahrens wurden zunächst fünf Proben filtriertes Schweineschmalz, dem Vorgange von Polenske folgend, genau in der beschriebenen Weise unter Einleiten eines sorgfältig getrockneten Kohlensäurestromes im Glycerinwassertrockenschrank 6 Stunden auf $100-101^{\circ}$ erhitzt und so vollständig von etwa vorhandenem Wasser befreit.

Während Polenske zu seinen Versuchen lediglich frisches amerikanisches Schmalz heranzog, wurden hier zu der Nachprüfung außer drei Proben amerikanischen Schmalzes je eine Probe holländisches und inländisches Schmalz verwendet. Die fünf Proben zeigten eine tadellose äußere Beschaffenheit, und lagen die ermittelten chemischen Konstanten in normalen Grenzen.

Um nun zunächst die Trübungstemperatur bei Zusatz ansteigender Mengen Wasser festzustellen, wurden den einzelnen filtrierten und entwässerten Proben 0,15 bis $0,45 \%$ Wasser zugesetzt, und dieses darin durch viermaliges Erhitzen auf $95^{\circ}$ bis $97^{\circ}$ unter beständigem Umschütteln gelöst. Das Lösen des Wassers und die Be-

1) Jetzt Assistent am Chemischen Laboratorium der Auslandsfleischbeschaustelle in Frankfurt a. M.

2) Arbeiten a. d. Kaiserl. Gesundheitsamte 1907, 25, 505; diese Zeitschrift 1907, 13, 754. N. 08 . 
stimmung der Trübungstemperatur erfolgte genau in der von Pole $\mathrm{n}$ s ke beschriebenen Weise. Die Trübungstemperatur war nach einiger Übung sehr scharf zu erkennen; Ablesungen, die bei derselben Probe häufiger nach einiger Zeit wiederholt wurden, ergaben gar keine oder nur ganz geringe Abweichungen von den zuerst ermittelten Zahlen.

Die hier gefundenen Zahlen sind in der nachstehenden Tabelle zusammengestellt.

Tabelle I.

Trübungstemperaturen $\left({ }^{\circ}\right.$ C) bei filtrierten Schmalzproben.

\begin{tabular}{|c|c|c|c|c|c|c|c|c|c|}
\hline \multirow{2}{*}{$\begin{array}{c}\text { I } \\
\text { Wasser- } \\
\text { gehalt } \\
\% \\
\%\end{array}$} & \multicolumn{5}{|c|}{$\begin{array}{c}\text { II } \\
\text { Trübungstemperaturen der Sehmalz- } \\
\text { proben }\end{array}$} & \multirow{2}{*}{$\begin{array}{c}\text { III } \\
\text { Mittiere } \\
\text { Trubungs- } \\
\text { temperatur }\end{array}$} & \multirow{2}{*}{$\begin{array}{c}\text { IV } \\
\text { Tempera- } \\
\text { turunter- } \\
\text { schiede }\end{array}$} & \multirow{2}{*}{$\begin{array}{c}\mathrm{V} \\
\text { Mittlere } \\
\text { Tribungugs- } \\
\text { temperatur } \\
\text { naeh } \\
\text { Polenske }\end{array}$} & \multirow{2}{*}{$\begin{array}{c}\text { VI } \\
\text { Tempera- } \\
\text { turunter- } \\
\text { sehiede } \\
\text { naeh } \\
\text { Polenske }\end{array}$} \\
\hline & No. 1 & No. 2 & No. 8 & No, 4 & No. 5 & & & & \\
\hline 0,45 & 95 & 95 & 94 & 95 & 97 & 95,2 & & 95,5 & \\
\hline 0,40 & 90 & 92 & 89 & 90 & 93 & 90,8 & $\begin{array}{l}\text {, } \\
5,8\end{array}$ & 90,8 & 5,8 \\
\hline 0,35 & 84 & 85 & 83 & 85 & 88 & 85,0 & $\begin{array}{l}, \circ \\
9,2\end{array}$ & 85,0 & 9.8 \\
\hline 0,30 & 74 & 76 & 74 & 77 & 78 & 75,8 & $\begin{array}{r}8,2 \\
11,2\end{array}$ & 75,2 & 10.7 \\
\hline 0,25 & 64 & 65 & 63 & 63 & 68 & 64,6 & $\begin{array}{l}11,4 \\
11,4\end{array}$ & 64,5 & 11,5 \\
\hline 0,20 & 52 & 54 & 51 & 53 & 56 & 58,2 & $\begin{array}{l}11,4 \\
12,0\end{array}$ & 53,0 & $\begin{array}{l}11,4 \\
12,5\end{array}$ \\
\hline 0,15 & 41 & 41 & 40 & 41 & 43 & 41,2 & & 40,5 & \\
\hline
\end{tabular}

Die Proben No. 1-3 waren amerikanische Schmalze, Probe No. 4 holländisches und die Probe No. 5 deutsches Schmalz. In Spalte V und VI sind die von Polenske gefundenen Trübungstemperaturen und Temperaturunterschiede angegeben. Aus einem Vergleich der Zahlen in Spalte III und IV mit den von Polenske gefundenen geht hervor, daß die hier gefundenen Zahlen auch bei holländischem und inländischem Schmalze nur wenig von den durch Polenske ermittelten Werten abweichen; für die Beurteilung eines Schmalzes spielen diese geringen Abweichungen in der Praxis keine Rolle. Die vorstehenden Zahlen sind bei filtrierten Schmalzen ermittelt worden. Um nun festzustellen, ob nach dem Verfahren der Wassergehalt auch bei Handelsschmalzen, die häufig etwas trübe schmelzen, annähernd genau festgestellt werden kann, wurden zu vier Proben Handelsschmalz, das in der eingangs beschriebenen Weise entwässert war, von dritter Seite wechselnde Mengen Wasser zugesetzt. Mit Absicht waren zu diesen Versuchen Schmalze ausgewählt, die nicht ganz klar schmolzen. Die Untersuchung dieser vier Schmalze, von denen die ersten drei aus Amerika, das vierte aus Holland stammte, lieferte folgende Ergebnisse:

Tabelle II.

Wasserbestimmang in nicht filtrierten Schmalzen.

\begin{tabular}{c|c|c}
\hline Gefundene Menge Wasser & Zugesetzte Menge Wasser & $\begin{array}{c}\text { Differenz zwischen gefundenem } \\
\text { und zugesetztem Wasser }\end{array}$ \\
\hline $0,379 \%$ & $0,380 \%$ & $-0,001 \%$ \\
$0,284 \%$ & $0,281 \%$ & $+0,003 \%$ \\
$0,179 \%$ & $0,162 \%$ & $+0,017 \%$ \\
0,420, & $0,440 \%$ & $-0,020 \%$
\end{tabular}


Es geht hieraus hervor, daß nach dem vorliegenden Verfahren der Wassergehalt auch bei etwas trübe schmelzenden Handelsschmalzen sehr genau ermittelt werden kann. Um die eintretende Trübung hier jedoch besser beobachten zu können, wurde neben der fraglichen Probe eine zweite Probe wäbrend des ganzen Versuches auf etwa $95^{\circ}$ erwärmt; es ließ sich so durch Vergleich der beiden Proben der Eintritt der Trübungstemperatur schärfer erkennen.

Nachdem auch durch die vorstehenden Versuche die Brauchbarkeit des Verfahrens festgestellt war, wurden hier in den letzten Monaten eine größere Anzahl Schmalzproben auf ihren Wassergehalt geprüft. Die Untersuchung nimmt bei einiger Übung nor verhältnismäßig wenig Zeit in Anspruch. Von den untersuchten 142 Proben, von denen 119 aus Amerika und 23 aus Holland stammten, zeigten 139 Proben eine Trübungstemperatur von unter $40^{\circ}$, ihr Wassergehalt betrug also böchstens $0,15 \%$.

Die übrigen drei Proben, sämtlich amerikanischen Ursprungs, enthielten 0,20 , 0,215 und $0,30 \%$ Wasser. Das Schmalz mit $0,20 \%$ Wasser war verdorben und wurde aus diesem Grunde hier beanstandet.

Von Polenske wird in Vorschlag gebracht, Schweineschmalz wegen seines Wassergehaltes erst dann zu beanstanden, wenn seine konstante Trübungstemperatur über $75^{\circ}$ liegt, d. h. wenn es mehr als $0,30^{\circ} \%$ Wasser enthält. Nach den hier gefundenen Zahlen enthielt von den untersuchten 142 Proben keine über $0,30 \%$ Wasser, nur drei Proben zeigten einen höheren Wassergehalt wie $0,15 \%$, es dürfte demnach hieraus hervorgehen, daß der Wassergehalt sowohl bei amerikanischen wie auch holländischen einwandfreien Schmalzen nur in seltenen Fällen $0,2 \%$ um ein geringes übersteigt.

Bei Bestimmungen des Wassergehaltes in Schweineschmalz, die in fräheren Jahren hier nach der antlich vorgeschriebenen Methode ausgeführt wurden, sind nachstehende Zahlen, die für die Beurteilung der vorliegenden Frage ein gewisses Interesse haben, für den Wassergehalt gefunden worden:

$\begin{array}{lccccc}\text { Anzahl der Proben } & 2 & 1 & 1 & 1 & 3 \\ \text { Wassergehalt } & 0,30 & 0,84 & 0,88 & 1,00 & 1,10 \%\end{array}$

Es mag hierbei erwähnt werden, daß im allgemeinen hier früher in Befolgung der amtlichen Anweisung zur chemischen Untersuchung von Fetten und Käsen, Absatz III, B. 1, Wasserbestimmungen nur bei den Schmalzen ausgeführt wurden, die beim Erwärmen trübe schmolzen, und bei denen die Gegenwart von Wasser zu erkennen war.

$$
\text { II. Talg. }
$$

Von den tierischen Fetten enthält außer dem Schweineschmalz auch der Talg bisweilen mehr oder minder große Mengen Wasser; beispielsweise wurde in den letzten Jahren hier in acht Proben Talg ein Wassergehalt zwischen 0,28-2,44\% gefunden.

Die Einfachheit, rasche Ausführung und die dabei trotzdem erzielte große Genauigkeit des vorstehend besprochenen Verfahrens zur Bestimmung des Wassergehaltes in Schweineschmalz legte es nun nahe, Versuche darüber anzustellen, ob das Verfahren in ähnlicher Weise auch bei Talg angewendet werden kann.

$\mathrm{Zu}$ diesem $Z$ wecke wurden zunächst mehrere Proben Handelstalg, die ungefähr denselben Erstarrungspunkt zeigten, und je eine Probe von selbst ausgelassenem Hammel- und Rindertalg, zum Teil nachdem erstere vorher filtriert waren, zum Teil ohne vorheriges Filtrieren, im Glycerin-Wassertrockenschrank unter Einleiten von Kohlen- 
säure bei $100-101^{\circ}$ so lange getrocknet, bis keine Gewichtsabnahme mehr erfolgte oder eine geringe Gewichtszunahme eintrat. In diesen so vorbereiteten Proben wurde in derselben Weise, wie bei den Schmalzproben beschrieben ist, $0,1-0,4 \%$ Wasser gelöst und dann die Trübungstemperatur bestimmt. Die Lösung des Wassers im Talge erfolgte weit schwieriger wie bei Schweineschmalz, und konnten nur bis zu $0,4 \%$ Wasser bei Wasserbadtemperatur in dem Talge gelöst werden. Als Trübungstemperatur wurde nicht die erste schwache Opaleszenz, sondern der etwas tiefer liegende Punkt angesehen, wo eine deutliche Trübung infolge Ausscheidung mikroskopiseh kleiner Wassertröpfchen wahrnehmbar war. Diese Trübung tritt bei Talg nicht immer mil derselben Deutlichkeit und nicht ebenso plötzlich ein wie bei Schmalz, sie ist aber auch bei solchen Talgproben, die an sich schon trübe schmelzen, auf böchstens $2^{0}$ deutlich zu erkennen. Ein Irrtum im Ablesen ist dann ausgeschlossen, wenn man darauf achtet, daß nach dem Eintritt der Trübungstemperatur die Trübung mit jedem weiteren Grade der Abkühlung stark zunehmen muß. Bei trübe schmelzenden Talgproben empfiehlt es sich, in ähnlicher Weise wie bei Schmalz besprochen ist, eine Vergleichsprobe zu verwenden.

Während nun bei Schweineschmalzproben, auch bei solchen verschiedener Herkunft und verschiedener äußerer Beschaffenheit, die Trübungstemperaturen bei demselben Gehalt an Wasser nur wenige Grade auseinanderliegen, zeigten die untersuchten Talgproben hierin, wie aus der folgenden Tabelle hervorgeht, ein ganz anderes Verhalten.

Tabelle III.

Trübungstemperaturen bei Talgproben.

\begin{tabular}{|c|c|c|c|c|c|c|c|c|c|c|}
\hline \multirow{3}{*}{$\begin{array}{c}\text { Wasser- } \\
\text { gehalt } \\
\%\end{array}$} & \multirow{2}{*}{\multicolumn{4}{|c|}{ Filtrierte Handelstalge }} & \multirow{2}{*}{\multicolumn{4}{|c|}{ Niwht flltrierte Handelstalge }} & \multicolumn{2}{|c|}{$\begin{array}{l}\text { Selbst ausgelassene } \\
\text { und filtrierte Talge }\end{array}$} \\
\hline & & & & & & & & & Rindertalg & Hammoltalg \\
\hline & No. 1 & No. 2 & No. 3 & No. 4 & No. 5 & No. 6 & No. 7 & No. 8 & No. 9 & No. 10 \\
\hline 0 & 350 & 350 & 360 & 350 & 350 & 380 & $96^{\circ}$ & 340 & 350 & 350 \\
\hline 0,10 & 350 & 350 & $36^{0}$ & 350 & 350 & $38^{0}$ & $36^{0}$ & 340 & $35^{0}$ & 350 \\
\hline 0,15 & 450 & $45^{\circ}$ & $46^{\circ}$ & $44: 9$ & $44^{0}$ & 500 & 540 & 390 & 380 & 420 \\
\hline 0,20 & 600 & 600 & 610 & 590 & 590 & $60^{\circ}$ & 670 & 520 & 410 & $55^{0}$ \\
\hline 0,25 & 700 & 680 & 720 & 710 & 690 & 690 & 820 & $61^{0}$ & 620 & 630 \\
\hline 0,30 & $80^{\circ}$ & 780 & 810 & $81^{0}$ & 810 & 790 & 930 & $70^{\circ}$ & $76^{0}$ & $70^{0}$ \\
\hline 0,35 & 870 & $86^{0}$ & $86^{\circ}$ & 870 & 900 & 850 & - & 790 & 870 & 790 \\
\hline 0,40 & 930 & $93^{\circ}$ & 940 & 930 & - & 910 & - & 880 & 940 & 900 \\
\hline
\end{tabular}

Nach der vorstehenden Zusammenstellung lag zwar bei den Proben No. 1-4, bei denen in filtrierten Proben Handelstalg ansteigende Mengen Wasser gelöst waren, bei gleichem Wassergehalt die Trübungstemperatur ziemlich in denselben Grenzen. Anders verhielten sich aber die Proben No. 5-8, bei denen Handelstalge vorlagen, die infolge der darin noch reichlich vorhandenen Gewebereste trübe schmolzen. Von diesen vier Proben lagen nur bei zweien (No. 5 und 6) die Trübungstemperaturen einigermaben innerhalb der Grenzen wie bei den filtrierten Talgproben, wohingegen bei den beiden anderen Proben hiervon ganz abweichende Trübungstemperaturen beobachtet wurden. Auch zwei selbst ausgelassene und vor Zusatz des Wassers ge- 
trocknete und klar filtrierte Proben Talg, und zwar ein Rinder- und ein Hammeltalg, die wasserfrei sich beide bei $35^{0}$ trübten, zeigten bei Zusatz derselben Mengen Wasser weit voneinander abweichende Trübungstemperaturen.

Aus diesen Versuchen geht nun herror, daß das von Polenske ausgearbeitete Verfahren zur Bestimmung des Wassergehaltes im Schweineschmalz auf Talg nicht ohne weiteres übertragen werden kann, daß vielmehr sowohl Rinds- und Hammeltalg, wie auch träbe schmelzende Handelstalge bei demselben Wassergehalt zum Teil recht weit voneinander abweichende Trübungstemperaturen zeigen.

Andererseits hat sich das Verfahren zur Bestimmung des Wassergehaltes im Schweineschmalz sehr gut bewährt. $E_{s}$ ist, um dieses nochmals hervorzuheben, leicht und schnell ausführbar und liefert dabei genauere Werte, wie das viel umständlichere und zeitraubendere Verfahren der amtlichen "Anweisung für die chemische Untersuchung von Fetten und Käsen“. Da eine Grenzzahl für den böchstzulässigen Wassergehalt im Schweineschmalz bislang nicht aufgestellt ist, schlägt $\mathrm{Pol}$ enske unter Berücksichtigung des bislang in besseren Marken amerikanischen Schweineschmalzes beobachteten Wassergehaltes vor, Schweineschmalz seines Wassergehaltes wegen erst dann zu beanstanden, wenn seine konstante Trübungstemperatur über $75^{0}$ liegt, d. $h$. wenn es mehr als $0,3 \%$ Wasser enthält.

Wie nun bereits vorher ausgeführt wurde, enthielten von den hier nach dem Verfahren von Polen ske untersuchten Proben Schmalz nur einige einen etwas höheren Wassergehalt wie $0,2 \%$, der aber in keinem Falle $0,3 \%$ überstieg, soda $\mathrm{B}$ hiernach der von Polenske vorgeschlagene höchstzulässige Grenzwert von $0,3 \%$ Wasser für den Fabrikanten sicherlich keine Härte bedeutet,

\title{
Über den Nachweis von Saponin.
}

\author{
Von
}

\section{Joh. Rühle.}

Mitteilung aus dem Königlichen chemischen Laboratorinm der Auslandsfleischbeschaustelle zu stettin.

Der Nachweis eines Zusatzes von schaumbildenden Mitteln zu schäumenden Getränken, wie Limonaden und Bier, scheint nach dem wenigen, das sich hierüber in der Literatur vorfindet, zu urteilen, zurzeit noch nicht mit völliger Sicherheit möglich zu sein. Nach Frehse ${ }^{1}$ ) wird Saponin in Brauselimonaden nachgewiesen, indem man diese möglichst zur Trockene eindampft und den Rückstand mit Essigäther auszieht. Nach dem Verdunsten der erhaltenen Lösung hinterbleibt bei Anwesenheit von Saponin ein Rückstand, der mit konzentrierter Schwefelsäure Rotviolettfärbung gibt und beim Kochen mit verdünnter Salzsäure in Sapogenin übergeführt wird unter Entbindung eines Geruches nach Cedernholz. In Champagnerlim on aden hat ferner Frehse ${ }^{2}$ ) Glycyrrhyzin beobachtet, das mit konzentrierter Schwefelsäure dieselbe Farbenreaktion wie Saponin gibt. Bei einer Nachprüfung des von Frehse für den Nachweis von Saponin angegebenen Verfahrens gelang es mir in

1) Journ. Pharm. Chim. 1899, [6] 10, 13-16; diese Zeitschrift 1899, 2, 938-939.

2) Journ. Pharm. Chim. 1899, [6] 10, 347-348; diese Zeitschrift 1900, 3, 365. 